Canadian

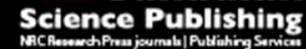

Canadian Journal of Chemistry Revue canadienne de chimie

\title{
Molecular Dynamics Screening For New Kinetic Inhibitors Of Methane Hydrate
}

\begin{tabular}{|r|l|}
\hline Journal: & Canadian Journal of Chemistry \\
\hline Manuscript ID: & cjc-2015-0003.R1 \\
\hline Danuscript Type: & Article \\
\hline Complete List of Authors: & $\begin{array}{l}\text { Oluwunmi, Paul; University of Warwick, Centre for Scientific Computing } \\
\text { Finney, Aaron; University of Warwick, Chemistry; University of Warwick, } \\
\text { Centre for Scientific Computing } \\
\text { Rodger, Mark; University of Warwick, Centre for Scientific Computing; } \\
\text { University of Warwick, Chemistry }\end{array}$ \\
\hline Keyword: & $\begin{array}{l}\text { hydrates, molecular dynamics, kinetic inhibition, low dosage inhibitors, } \\
\text { nucleation }\end{array}$ \\
\hline
\end{tabular}

SCHOLARONE ${ }^{\text {m }}$

Manuscripts 


\title{
Molecular Dynamics Screening For New Kinetic
}

\section{Inhibitors Of Methane Hydrate}

\author{
Paul A. Oluwunmi, Aaron R. Finney, P. Mark Rodger* \\ Department of Chemistry and Centre for Scientific Computing, University of Warwick, \\ Coventry, CV4 7AL, U.K.
}

KEYWORDS Methane hydrate, gas hydrates, clathrates, molecular dynamics, kinetic inhibitors, nucleation

ABSTRACT The development of polymeric and oligomeric chemical additives that can control the nucleation and growth of gas hydrates remains a topic of major research interest, with important implications for energy security and the environment. In this paper we present a molecular dynamics study of eight different oligomeric compounds that have been proposed as potential kinetic inhibitors for methane hydrate. The results show that statistically significant variations in hydrate formation, induced by the chemical additive, can be observed within a relatively modest series of molecular dynamics simulations, thus opening the way for computational screening for optimal additives to control hydrate formation. One amino acid oligomer, asparagine, was found to be more active than a number of synthetic inhibitors, including PVCap. 


\section{Introduction}

Clathrate hydrates are crystalline solids in which a water lattice (the "host") creates cages entrap another type of molecule (the "guest"). Many different compounds that can form clathrate hydrates have been documented, ${ }^{1-3}$ but for practical applications the most important the components of natural gas, particularly methane; hence these solids are often termed "gas hydrates", or simply "hydrates". At moderate to high pressures, gas hydrates will form at temperatures that can be significantly above the $273 \mathrm{~K}$. Such conditions are common geologically, in permafrost and at and below the sea floor, and have led to the formation of vast natural deposits of gas hydrates. ${ }^{4}$ These conditions are also common in oil and gas wells and pipelines. When this happens, the solid hydrate formation can result in blockages, leading to adverse economic and even environmental consequences. As a result, understanding how current inhibition methods work, ${ }^{5-7}$ and finding ways of improving them, ${ }^{8-10}$ remains a major field of research. $^{11}$

Chemical inhibition of hydrate formation can be achieved in two fundamentally different ways: thermodynamic of kinetic. Thermodynamic inhibitors, such as methanol or ethylene glycol, ${ }^{12}$ shift the three-phase equilibrium boundary to more extreme conditions, and hence make hydrate formation less likely. These are predictable and reliable, but often have to be employed in very large volumes, and so can be very expensive for use in offshore oil and gas production. Kinetic inhibitors act by delaying the onset of solid formation (i.e. delaying crystal nucleation), or by slowing the subsequent crystal growth to retain a fluid suspension. In general, the kinetic inhibitors can be used at much lower concentrations than the thermodynamic inhibitors, which makes the development of more active kinetic inhibitors-or "low dosage hydrate inhibitors" (LDHIs) — a major target for research in this area. 
Finding more active LDHIs is not a simple task. A great deal of effort has gone into screening programs $^{11,13}$ and promising new lead compounds are being identified, ${ }^{8,14,15}$ but in general the improvements are incremental. To a large extent, this is probably due to the lack of a clear mechanistic understanding of how LDHIs work. Several mechanisms have been proposed. The most prevalent is irreversible binding of the LDHI to the surface of small hydrate crystals and pre-critical clusters, ${ }^{2}$ in a manner similar to that proposed for antifreeze proteins. It is likely that such LDHIs actually promote hydrate nucleation, since the molecular recognition that favors surface binding should also create seed sites for nucleation, but prevent the resultant nanocrystals from growing to a size at which they can be observed; thus they would appear to delay nucleation. ${ }^{16}$. Reversible binding to the hydrate surface, or more correctly within the hydrate/water interfacial zone, has also been suggested. In this case, modifications to the water structure have been observed to destabilize small hydrate clusters and hence increase induction times to nucleation. ${ }^{17}$ Modifying water structure more generally has also been suggested, ${ }^{18}$ but without some mechanism for targeting regions of hydrate growth, this is simply a mechanism for changing water activity and is more properly classed as a thermodynamic effect. Preferential solvation of the inhibitor by guest molecules may also play a role in determining the LDHI activity. In reality, it is likely that a number of mechanisms are viable, and that the success of synergistic inhibitor blends ${ }^{19,20}$ arises because the different inhibitors within the blend interact with a number of different hydrate nucleation and growth mechanisms.

A common thread through all these mechanisms is that they are defined on a molecular length scale, and as such a definitive identification of the LDHI mechanism(s) requires a greater understanding of the molecular energetics and dynamics involved. Various experimental techniques have been used to characterize the molecular behavior of inhibitors, including NMR 
to monitor hydrate growth in the presence of several LDHIs ${ }^{7}$ and to study catastrophic growth with PVP and PVCap ${ }^{21}$, in situ Raman spectroscopy to show that PVCap reduces the rate of large cage formation during the early stages of hydrate formation ${ }^{22}$ and small angle neutron scattering to quantify the extent of surface adsorption of PVP on hydrates. ${ }^{23,24}$ Molecular modeling has also proved to be very powerful in elucidating mechanisms for hydrate growth and inhibition. There are now a number of different groups that have developed methods to model hydrate nucleation repeatably ${ }^{25-29}$ and growth ${ }^{30,31}$. Far fewer simulations have been performed with inhibitors, ${ }^{16,32,33}$ but these have established the potential for using molecular dynamics simulations to predict the effect of polymeric additives on the early stages of hydrate formation and growth. In the present paper we present an extensive molecular dynamics study of methane hydrate nucleation and growth in the presence of eight different proposed LDHIs, including both synthetic and bio-inspired chemical motifs. We show that statistically significant affects on initial hydrate growth can be demonstrated from relatively modest scale simulations, and also identify some unexpected solubility effects associated one of the amino-acid based polymers that is predicted to be amongst the most active.

\section{Methods}

Simulation details. All molecular dynamics simulations were performed in the NVT ensemble using DL_CLASSIC version $1.9^{34}$ with a timestep of 0.001 ps. Orthorhombic periodic boundary conditions were used throughout and temperature controlled using a Nosè-Hoover thermostat with a relaxation time of 0.5 ps. ${ }^{35,36}$ A rigid geometry for the water molecule was imposed using SHAKE. ${ }^{37}$ Van der Waals forces were truncated at $12 \AA$, and electrostatic interactions evaluated using the Smoothed Particle Mesh Ewald method (SPME). ${ }^{38}$ 
Force fields. The force fields used for this project are same to the ones used in many previous studies ${ }^{39-42} 16,17$. The SPC water force field was used to model the water-water interaction [39]. We note that although SPC water seriously underestimates the freezing point of water, it does a surprisingly good job with methane hydrate stability. ${ }^{43}$ Methane and chemical additives were modeled with the CHARMM 27 force field using united atoms for nonpolar hydrogens, ${ }^{44}$ and Lorenz-Berthelot mixing rules used for cross-interactions with the water.

Chemical "inhibitors". Eight different additives were considered in this study, with the chemical compositions shown in Scheme 1. In each case we have modeled an octomer, which gives molecular weights comparable to those found to be most active for PVP and PVCap ${ }^{45}$. We shall refer to them throughout as "kinetic inhibitors", or simply as "inhibitors", though we note that their ability to inhibit methane hydrate formation is postulated rather than proven in many cases.

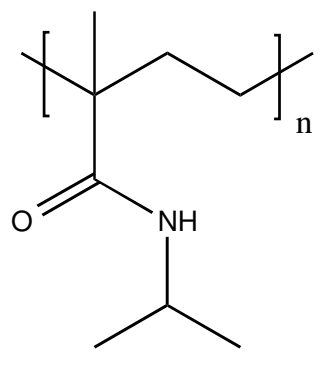

I

Pimam

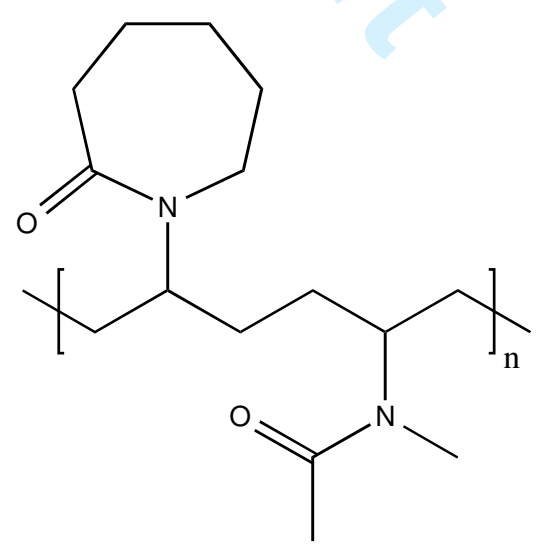

II Pvcap-vima

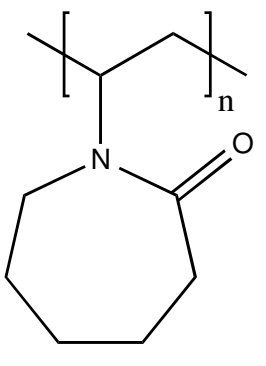

III PVCap 

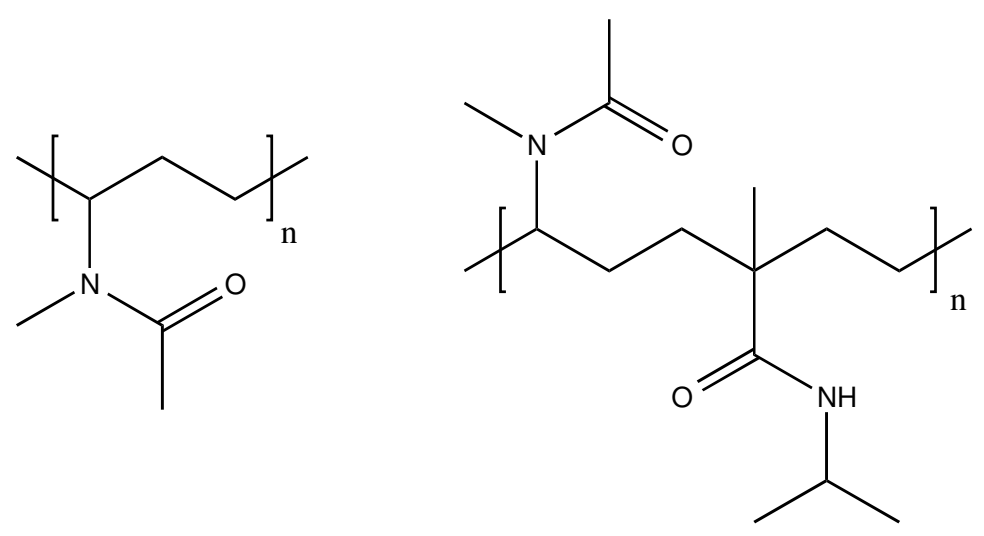

V

Pvima-imam

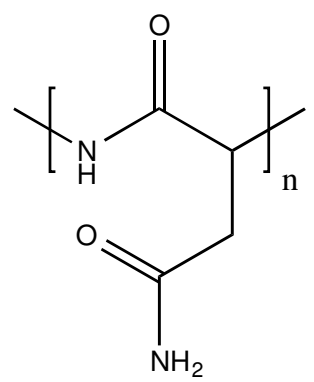

VII

ASN

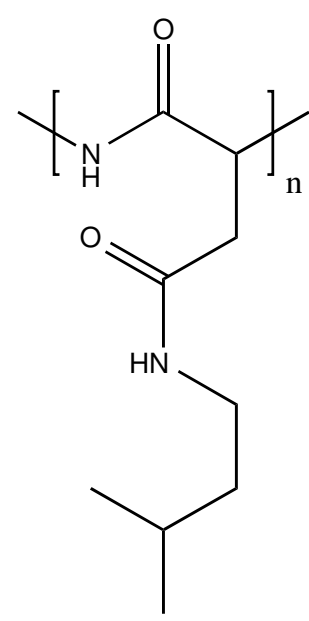

VI

ASN5

Scheme 1: chemical structures of the inhibitors. Atactic octomers were used in all cases, and the copolymers were random copolymers.

Construction of initial configurations. The initial configuration water/methane configuration was taken from our original simulations of methane hydrate nucleation within a thin film of water surrounded by methane gas. ${ }^{25}$ Two different configurations have been used in the current study: the first was taken from the very start of our original simulation and contained no significant hydrate content; the second was taken after about $5 \mathrm{~ns}$, by which stage a significant cluster of hydrate water had formed. Both systems contained 1656 ware molecules and 188 methane molecules with the dimensions of the respective simulation boxes being $3.94 \times 3.60 \times 11.70 \mathrm{~nm}(t=0)$ and $3.42 \times 3.04 \times 15.56 \mathrm{~nm}(t=5 \mathrm{~ns})$, where the $3 \mathrm{rd}$ dimension 
(i.e. $z$ ) is perpendicular to the water/gas interfaces. Images of these two starting configurations are provided as supplementary information (Figure S1).

The two initial configurations described above were simulated without inhibitors as control systems. To allow repeat simulations, and hence a statistical analysis of any hydrate growth during the simulations, four analogous starting configurations were created, that differed in the arrangement of the methane molecules and in the initial velocities assigned to all atoms, but retained exactly the same water configuration (and hence the same implicit hydrate structure to begin with). This was achieved by conducting an $N V T$ simulation in which the water was immobilized but the methane molecules allowed to move, and dumping out a new configuration every 5 ps. Our previous studies of pDMAEMA had shown that this provided trajectories with very different hydrate growth patterns over the subsequent 4 ns. ${ }^{16,33}$

Inhibited systems were created from these water film configurations using the same protocol we have used in previous hydrate inhibitor simulation studies: ${ }^{16}$ the inhibitor was introduced into the methane gas at a distance of at least $5 \AA$ away from the water film, and methane molecules that overlapped with the inhibitor were removed; the water was then immobilized and a short (ca. 5 ps) NVT simulation conducted to allow the methane and inhibitor geometries to relax to remove any stress induced by the insertion process; these were then used as the starting configuration for subsequent production runs. The rationale behind this protocol is to ensure that the insertion of the additive did not disturb the molecular structure of the water film, and hence to ensure the inhibited and control simulations started from as similar a water state as possible.

Notation. Uninhibited simulations will be denoted C (Control), and inhibited simulations by the appropriate roman numeral in scheme 1 to indicate which inhibitor is present. The starting water film will be indicated by the subscript 0 or 50 (to indicate the size of the largest initial 
hydrate cluster). Where necessary, the individual repeat simulations will be denoted by an additional subscript: $\mathrm{a}, \mathrm{b}, \mathrm{c}$ or $\mathrm{d}$.

\section{Results and discussion}

Previous studies have shown that it is possible to simulate nucleation of methane hydrate directly, ${ }^{27,32}$ but that long simulation times may be necessary. Likewise, multiple simulations of the effect of chemical additives have been reported, ${ }^{16,17,33}$ but those simulations were initiated with a substantial hydrate cluster ( $c$ a. 300 water molecules) already present. It is of interest to know to what extent chemical inhibition can be simulated without seeding the simulation, and under what conditions this can be done within simulations of a few nanoseconds, since this timescale would make computational screening with molecular dynamics viable. We have therefore conducted a set of simulations designed to identify the conditions under which rapid hydrate formation may be expected using the current potentials. Two initial configurations were constructed, as described in the methods section: one contained no substantial hydrate cluster and the other a cluster with about 50 water molecules. Both systems were simulated at temperatures in the range $200-250 \mathrm{~K}$. These simulations formed the control systems for subsequent inhibited simulations. The aim of these simulations was to establish which temperature and which systems favored methane hydrate growth.

\section{Control Simulations}

Several methods were used to monitor for hydrate growth. Experimentally, the onset of crystallization can be seen as a temperature spike due to the enthalpy of fusion, or as a pressure drop due to the encapsulation of methane in the clathrate. The former is seen in constant temperature MD simulations as a decrease in the potential energy and has been used within this study to monitor for hydrate growth; pressure drops are not so easy to see in simulations, as the 
small size of MD simulations means that the innate pressure fluctuations are much larger than the pressure drop that would be caused by the formation of a small hydrate cluster. As an additional probe for hydrate formation we have used the local phase assignment that we developed previously; ${ }^{46-48}$ this enables individual water molecules to be identified according to whether their local environment matches that of the crystalline hydrate, ice, or liquid water; the number of such hydrate-like water molecules gives a convenient measure of hydrate content. Radial distribution functions for water molecules with hydrate or liquid local phase are given in Figure 1, and compared with RDFs for bulk methane hydrate and bulk water. It is clear that the local phase RDFs reproduce the key features of the respective bulk phases; given that the hydrate waters represent just $20 \%$ of the water within these simulations, it is not surprising that the peaks in this case a broader than for bulk methane hydrate, however the peak locations of the bulk system are clearly reproduced. For water molecules with liquid local phase, which constitutes about $70 \%$ of the water in the film, the RDF is essentially indistinguishable from that for pure liquid water.

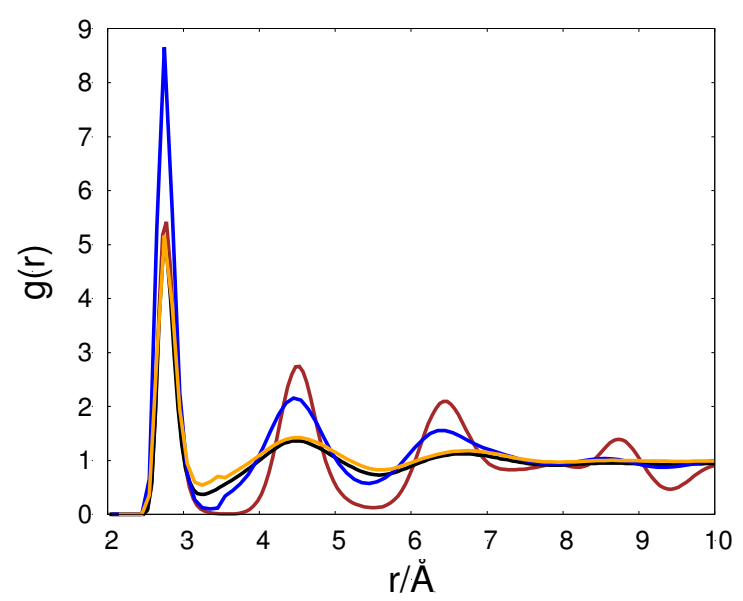

Figure 1. Radial distribution functions for water molecules in different environments ("local phases") at $240 \mathrm{~K}$ : hydrate-hydrate within the water film, brown; liquid-liquid within the water film, black; water within build methane hydrate, brown; water within bulk liquid water, orange 
The time-evolution of the hydrate content and of the total potential energy in e $\mathrm{C}_{0}$ simulations at different temperatures is presented in Figure 2. The behavior for $\mathrm{C}_{50}$ was quantitatively similar. At $200 \mathrm{~K}$ and $230 \mathrm{~K}$ there is a clear increase in hydrate content, correlated with a decrease in total potential energy, indicating sustained hydrate growth during the simulations. In contrast, there is an increase in potential energy and decrease in hydrate content during the simulation at $250 \mathrm{~K}$, indicating that the hydrate is unstable under these conditions (with these potentials). At the same time, there is a loss of methane from the water film at $250 \mathrm{~K}$ that is not seen at the other temperatures (Supplementary Information, Figure S2). A temperature of $240 \mathrm{~K}$ (1 bar) appears to favor hydrate growth, but only slightly: there is slight decrease in potential energy over most of the simulation, albeit after an initial rapid rise, and the hydrate content increases slightly across the simulation. Radial distribution functions (Supplementary Information, Figure S3) indicate that some hydrate structure persists at temperatures of $240 \mathrm{~K}$ and below, but is not present at $250 \mathrm{~K}$. Based on this data, a convenient measure of the overall growth (or degradation) of hydrate during a simulation can be obtained by calculating the difference in the average number of hydrate water molecules in the system during the first and last 0.1 ns of each simulation

$$
\Delta N=\left\langle N_{\text {hyd }}\right\rangle_{3.9 \mathrm{~ns}<t<4.0 \mathrm{~ns}}-\left\langle N_{\text {hyd }}\right\rangle_{0<t<0.1 \mathrm{~ns}}
$$

Values of $\Delta N$ for the control simulations are shown in Figure 3. Statistical $t$-tests indicate that, at the $95 \%$ significance level: (1) there is no significant difference between the $\mathrm{C}_{0}$ and $\mathrm{C}_{50}$ systems; (2) there is significant growth in hydrate content for temperatures less than $240 \mathrm{~K}$, and melting above $240 \mathrm{~K}$, but that any changes in hydrate content during the simulations at $240 \mathrm{~K}$ cannot be deemed to be significant. 

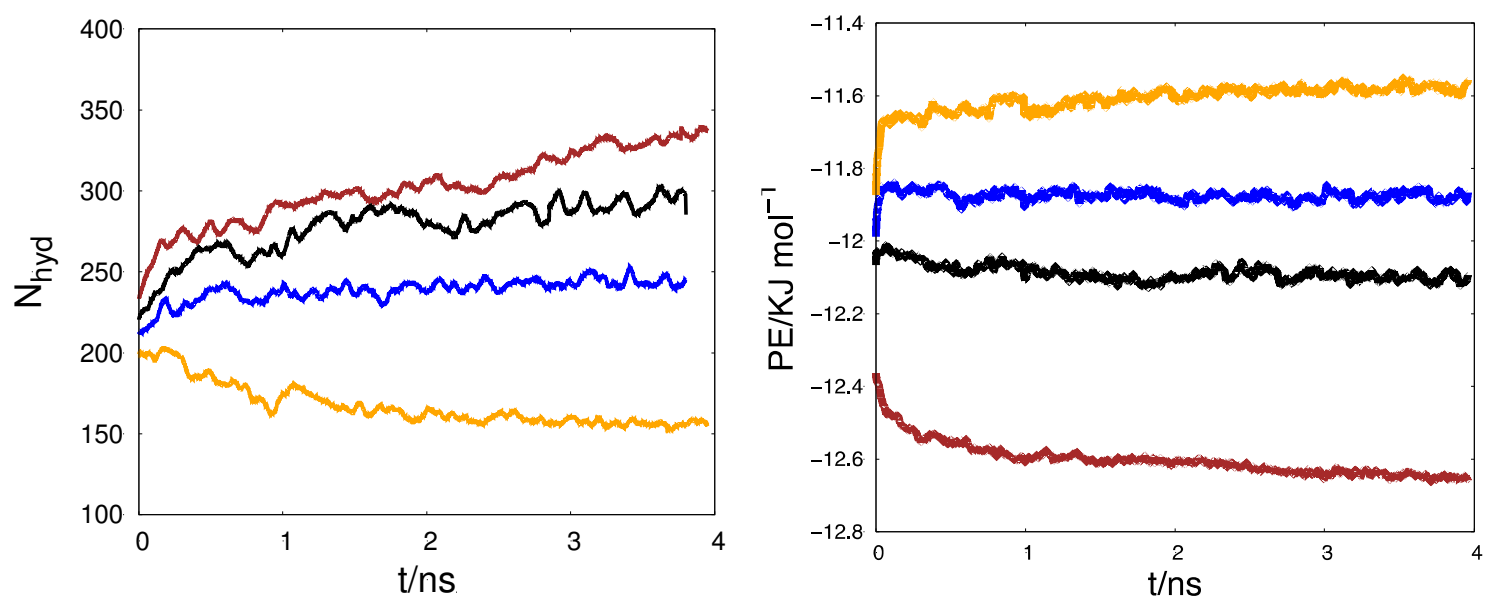

Figure 2. Time dependence of the hydrate content (left) and potential energy (right) $\mathrm{n}$ the $\mathrm{C}_{0}$ simulations at $200 \mathrm{~K}$ (brown), $230 \mathrm{~K}$ (black), $240 \mathrm{~K}$ (dark blue) and $250 \mathrm{~K}$ (dark yellow). $N_{\text {hyd }}$ is the number of water molecules with hydrate local phase, where the total number of water molecules in the simulation is 1656.

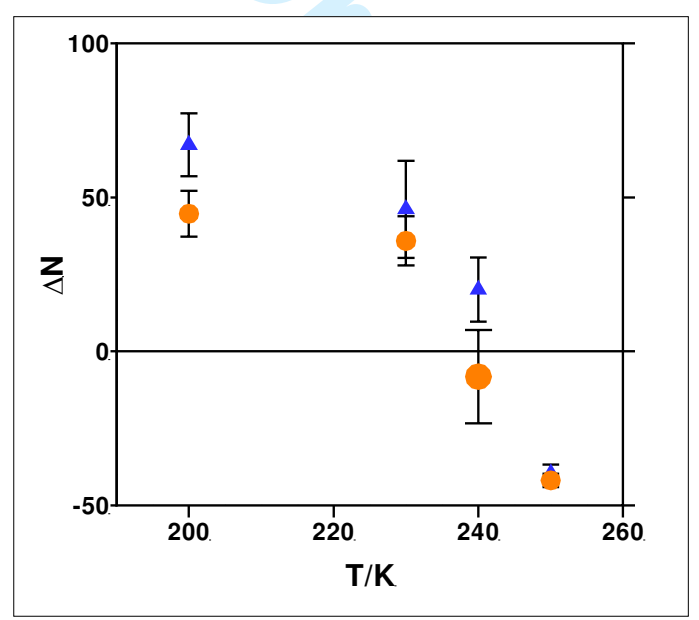

Figure 3. Change in hydrate content over time for the $\mathrm{C}_{0}$ (blue triangles) and $\mathrm{C}_{50}$ (orange circles) systems. $\Delta N$ is defined in Equation (1).

Where hydrate growth is observed, closer analysis also shows that the hydrate-like water molecules are aggregating, although the dynamics of cluster formation can be very varied. To probe this we have calculated the distribution of cluster sizes within $1 \mathrm{~ns}$ time-windows of each 
simulation; for this purpose we have defined two water molecules to be "bonded" if they both have hydrate local phase and are within $3.5 \AA$, and two water molecules belong to the same cluster if they are connected by an unbroken chain of "bonded" water molecules. As examples, cluster size distributions calculated from $\mathrm{C}_{0 \mathrm{a}}$ and $\mathrm{C}_{0 \mathrm{~b}}$ are depicted in Figure 4. Both show increases in the typical cluster sizes over the duration of the simulation, with both showing clusters with at least 150 water molecules by the end of the simulation; note that the $\mathrm{C}_{0}$ systems started with no significant hydrate clusters. However, the evolution of the clusters in these two simulations is substantially different, as should be expected for a stochastic process such as nucleation: in $\mathrm{C}_{0 \mathrm{a}}$ there is a broad distribution of cluster sizes, with a modal cluster size that increases steadily from 50 to 100 water molecules; in $\mathrm{C}_{0 \mathrm{~b}}$, however, larger clusters (up to 200 hydrate water molecules) form within $2 \mathrm{~ns}$, with evidence that they both grow and fragment during the last ns of the simulation.
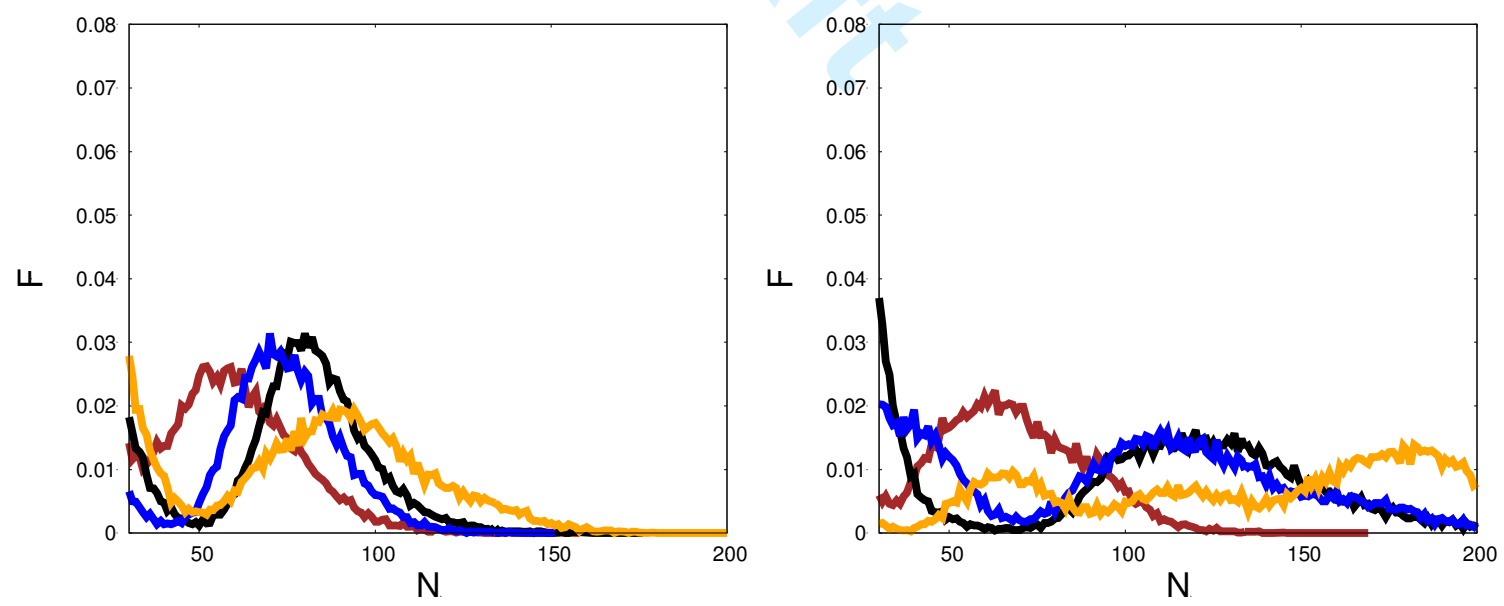

Figure 4. Cluster size distribution for $\mathrm{C}_{0 \mathrm{a}}$ and $\mathrm{C}_{0 \mathrm{~b}}$ at $230 \mathrm{~K}$ : 0-1 ns (brown); 1-2 ns (blue); 2-3 ns (black); 3-4 ns (yellow). 
We conclude that temperatures of $230-240 \mathrm{~K}$ are appropriate conditions under which to study the onset of hydrate formation with this model of methane hydrate, and hence are appropriate temperatures with which to examine the influence of potential inhibitors. We also note that the underlying dynamics of hydrate formation - or melting - is stochastic, and hence that multiple simulations are needed in order to draw significant conclusions about the factors which control the early stages of hydrate formation.

\section{Inhibited systems}

Eight different inhibitors were introduced into the initial configurations as described in Methods, and then simulations performed and monitored for the subsequent effect on hydrate formation. In the case of the two amino acids, both extended and $\alpha$-helical forms of the octomer were used (denoted by subscript "e" and "a", respectively). Results, in terms of the overall change in hydrate content during the simulation, are presented in Figure 5 . At $230 \mathrm{~K}$, most of the inhibitors show no significant from the control system, and no significant difference between the two initial water configurations. The one exception is VII, the amino acid asparagine, which in the $\alpha$-helical form produced significantly less hydrate growth than was found in the control system $(t$-test, $p=0.04)$, and essentially prevented any hydrate growth.
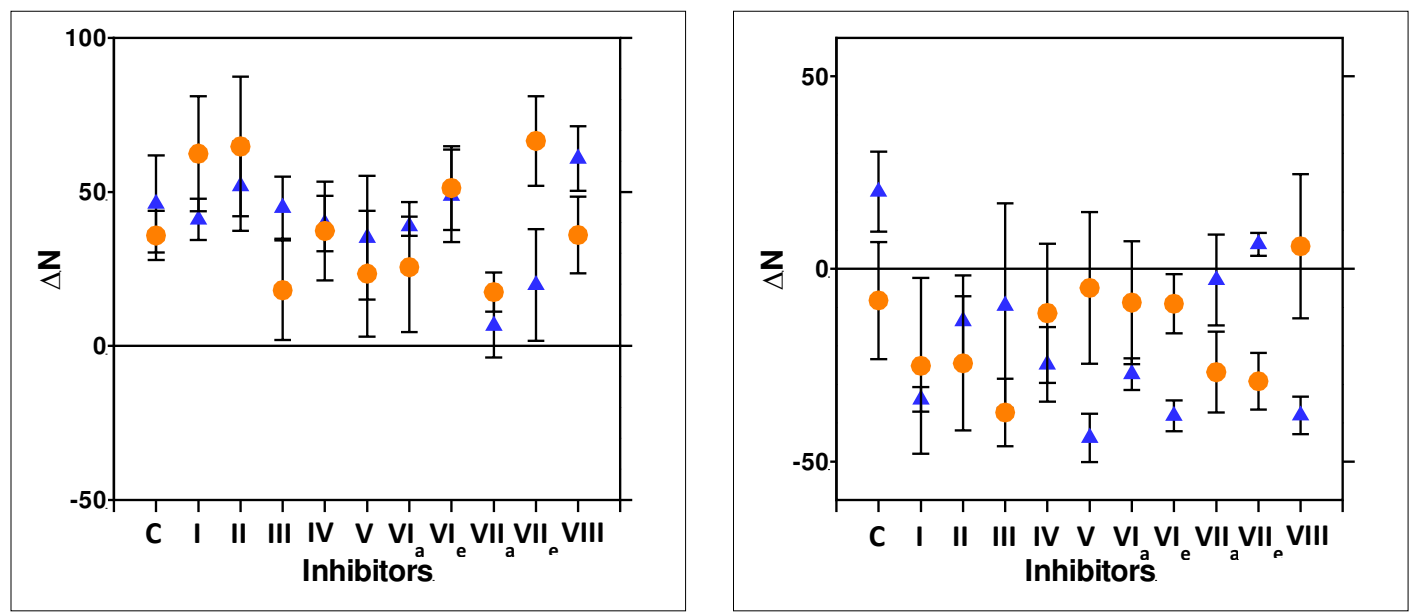
Figure 5. Change in hydrate content in the presence of inhibitors at $230 \mathrm{~K}$ (left) and $240 \mathrm{~K}$ (right): blue triangles are for simulations derived from the $\mathrm{C}_{0}$ initial configuration; orange circles are derived from the $\mathrm{C}_{50}$ initial configuration.

At $240 \mathrm{~K}$, the situation is more complex. There are now a number of systems that show significant activity as inhibitors, although the effect depends on the size of the initial hydrate cluster. In particular, for the series of simulations based on $\mathrm{C}_{0}$ (i.e. with no original hydrate cluster), inhibitors I, IV, V, VI and VIII give a highly significant $(p<0.01)$ reduction the hydrate content compared with the control, diminishing the hydrate content over time whereas the control shows some hydrate growth. However, none of the inhibitors give a significant improvement in the $\mathrm{C}_{50}$ series. We note that nucleation inhibitors should be affected by seeding the system, and this has been observed previously in PVP ${ }^{17}$. However, we also note that the data at $240 \mathrm{~K}$ typically showed large uncertainties which, on close inspection, result from quite different aggregation/decomposition behavior within each set of four equivalent simulations. Such behavior is to be expected under conditions where the thermodynamic driving force becomes weak and induction times for nucleation become long, and much larger statistical samples are likely to be needed in such cases.

The unique behavior of VII (asparagine) is also seen in its solubility. The distribution of inhibitors across the water film is shown in Figure 6. Most of the inhibitors show that the distribution is strongly focused on the water/methane interface. Such an effect is well known for large molecules in mixtures of large and small molecules ${ }^{49,50}$ and is largely due to entropic effects rather than the substantive mixed hydrophobic/hydrophilic interactions that are found in surfactants. Asparagine, however, is far more soluble, and the distribution clearly shifts into the liquid water film. It is interesting to note that in previous studies of pDMAEMA, interaction with 
the hydrate was found to be mediated through those monomer units that entered the water phase rather than those that favored the interfacial region. Thus it is likely that the greater solubility of asparagine is implicated in its more effective hydrate inhibition at $230 \mathrm{~K}$.
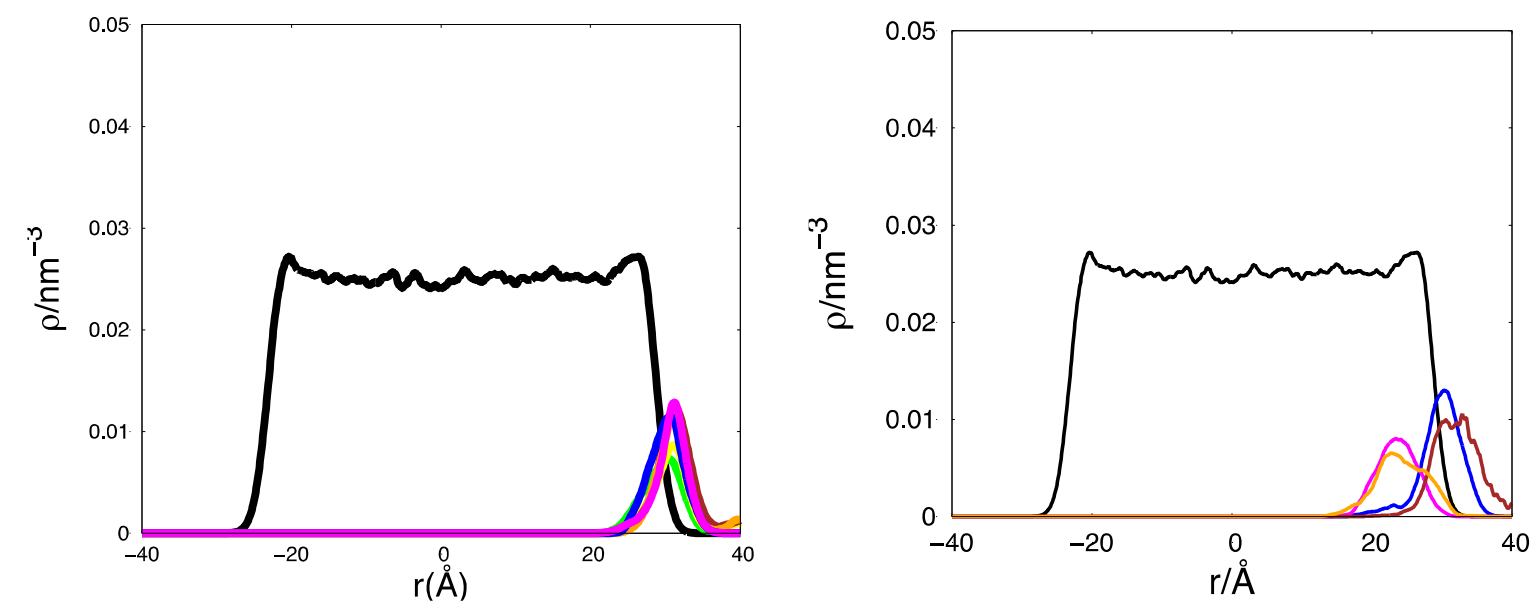

Figure 6. $z$-density of water and inhibitor for the $\mathrm{C}_{50}$ series of simulations. Water density is depicted in black, and clearly shows the location of the water film. Synthetic polymers are given on the left: I (blue); II (orange); III (brown); IV (yellow); V (magenta); VIII (green). Amino acid inhibitors are given on the right: $\mathbf{V I}_{\mathbf{a}}$ (blue); $\mathbf{V I} \mathbf{I}_{\mathbf{e}}$ (brown); $\mathbf{V I I}_{\mathbf{a}}$ (magenta); $\mathbf{V I I}_{\mathbf{e}}$ (orange)

\section{Conclusions}

In this paper we have presented the results of a series of molecular dynamics simulations designed to probe the influence of a series of potential inhibitors on the earliest stages of hydrate formation. Different series of simulations were performed starting with a small hydrate cluster (about 50 water molecules) or no hydrate cluster in order to test the effect of seeding hydrate formation; earlier simulations of hydrate inhibitors have seeded the simulation with hydrate clusters containing 200-300 water molecules. In the absence of any inhibitor simulations sustained hydrate growth was observed at temperatures below $240 \mathrm{~K}$ at $1 \mathrm{~atm}$, and the rate of growth appeared to be independent of the extent to which the simulation was initially seeded with a hydrate nanocluster. At temperatures above $250 \mathrm{~K}$ hydrate was consistently found to melt. The behavior at $240 \mathrm{~K}$ showed a mixture of behavior consistent with a weak thermodynamic 
driving force for hydrate formation and nucleation inhibition times that were comparable with the duration of the simulations.

Eight different potential inhibitors were examined by introducing the inhibitor into the gas phase and allowing it to diffuse to the water. Of these, six were common synthetic oligomers and two were amino acids. At $230 \mathrm{~K}$, only the asparagine was found to generate a statistically significant effect on hydrate growth, effectively suppressing any hydrate formation during the simulation; this was independent of the initial hydrate seed. Intriguingly, it was found that all inhibitors except asparagine adsorbed at the water/methane interface, while asparagine proved much more hydrophilic. Much more varied behavior was observed at $240 \mathrm{~K}$, with 5 of the inhibitors showing inhibitory activity, but with the results depending on the initial seeding of hydrate growth. We conclude that statistically significant variations in hydrate growth rates, induced by chemical additives, can be observed within molecular dynamics simulations of a relatively modest scale, and thus that computational screening for new hydrate inhibitors is now viable. To realize the full potential of such computational screening, however, work will be needed to identify the most appropriate force-fields for quantitatively accurate predictions under experimental conditions.

\section{ASSOCIATED CONTENT}

Figures depicting initial configurations, $\mathrm{z}$ density profiles for methane, and water methane RDFs. This material is available free of charge via the Internet at http://pubs.acs.org.

\section{AUTHOR INFORMATION}

\section{Corresponding Author}

* P.M. Rodger, p.m.rodger@warwick.ac.uk 


\section{Author Contributions}

PMR planned and oversaw the research and wrote the manuscript; ARF assisted in conducting the simulations; PAO conducted the simulations and analysis and helped write the manuscript; all three authors contributed to the design of the analysis and the interpretation of the data.

\section{ACKNOWLEDGMENT}

The authors wish to acknowledge the tremendous contribution toward understanding hydrate crystallization that ahs been made by Prof John Ripmeester; PMR is particularly grateful for many stimulating discussions over the years that have helped developed his understanding of these materials. Computer time was supplied by the Scientific Computing Research Technology Platform at Warwick University, by the MidPlus Regional Centre of Excellence for Computational Science, Engineering and Mathematics, under EPSRC grant EP/K000128/1

\section{REFERENCES}

(1) Lee, J. W.; Lu, H. L.; Moudrakovski, I. L.; Ratcliffe, C. I.; Ohmura, R.; Alavi, S.; Ripmeester, J. A. J. Phys. Chem. A 2011, 115, 1650.

(2) Sloan, E. D. Clathrate Hydrates of Natural Gases; 2nd ed.; Marcel Dekker, Inc.: New York, 1998.

(3) Brouwer, D. H.; Brouwer, E. B.; Maclaurin, G.; Lee, M.; Parks, D.; Ripmeester, J. A. Supramol. Chem. 1997, 8, 361.

(4) Milkov, A. V. Earth-Sci. Rev. 2004, 66, 183.

(5) Sharifi, H.; Ripmeester, J.; Walker, V. K.; Englezos, P. Fuel 2014, 117, 109.

(6) Yang, J. H.; Tohidi, B. Energy Fuels 2013, 27, 736.

(7) Daraboina, N.; Moudrakovski, I. L.; Ripmeester, J. A.; Walker, V. K.; Englezos, P. Fuel 2013, 105, 630.

(8) Sa, J. H.; Kwak, G. H.; Lee, B. R.; Park, D. H.; Han, K.; Lee, K. H. Sci Rep 2013, $3,2428$.

3154.

(9) Reyes, F. T.; Malins, E. L.; Becer, C. R.; Kelland, M. A. Energy Fuels 2013, 27,

(10) Cha, M.; Shin, K.; Kim, J.; Chang, D.; Seo, Y.; Lee, H.; Kang, S. P. Chem. Eng. Sci. 2013, 99, 184.

(11) Perrin, A.; Musa, O. M.; Steed, J. W. Chem. Soc. Rev. 2013, 42, 1996.

(12) Creek, J. L. Energy Fuels 2012, 26, 4112.

(13) Lone, A.; Kelland, M. A. Energy Fuels 2013, 27, 2536. 
(14) Sina, M.; Nazari, K.; Mohammad-Taheri, M.; Moradi, M. R. Chem. Eng. Technol. 2013, 36, 1117.

(15) Reyes, F. T.; Kelland, M. A. Energy Fuels 2013, 27, 3730.

(16) Hawtin, R. W.; Rodger, P. M. J. Mater. Chem. 2006, 16, 1934.

(17) Moon, C.; Hawtin, R. W.; Rodger, P. M. Faraday Discuss. 2007, 136, 367.

(18) Varma-Nair, M.; Costello, C. A.; Colle, K. S.; King, H. E. J. Appl. Polym. Sci. 2007, 103, 2642.

(19) Kelland, M. A.; Moi, N.; Howarth, M. Energy Fuels 2013, 27, 711.

(20) Daraboina, N.; Malmos, C.; von Solms, N. Fuel 2013, 108, 749.

(21) Cha, M.; Shin, K.; Seo, Y.; Shin, J. Y.; Kang, S. P. J. Phys. Chem. A 2013, 117, 13988.

(22) Hong, S. Y.; Jim, J. I.; Kim, J. H.; Lee, J. D. Energy Fuels 2012, 26, 7045.

(23) King, H. E.; Hutter, J. L.; Lin, M. Y.; Sun, T. J. Chem. Phys. 2000, 112, 2523.

(24) Hutter, J. L.; King, H. E.; Lin, M. Y. Macromolecules 2000, 33, 2670.

(25) Moon, C.; Taylor, P. C.; Rodger, P. M. J. Am. Chem. Soc. 2003, 125, 4706.

(26) Hawtin, R. W.; Quigley, D.; Rodger, P. M. Phys. Chem. Chem. Phys. 2008, 10, 4853.

(27) Walsh, M. R.; Koh, C. A.; Sloan, E. D.; Sum, A. K.; Wu, D. T. Science 2009, $326,1095$.

(28) Jacobson, L. C.; Hujo, W.; Molinero, V. J. Phys. Chem. B 2010, 114, 13796.

(29) Jimenez-Angeles, F.; Firoozabadi, A. J. Phys. Chem. C 2014, 118, 11310.

(30) Vatamanu, J.; Kusalik, P. G. J. Phys. Chem. B 2008, 112, 2399.

(31) Liang, S.; Kusalik, P. G. J. Phys. Chem. B 2010, 114, 9563.

(32) Moon, C.; Taylor, P. C.; Rodger, P. M. Can. J. Phys. 2003, 81, 451.

(33) Zhang, J. F.; Hawtin, R. W.; Yang, Y.; Nakagava, E.; Rivero, M.; Choi, S. K.; Rodger, P. M. J. Phys. Chem. B 2008, 112, 10608.

(34) Smith, W.; Todorov, I. T. Molecular Simulation 2006, 32, 935.

(35) Nose, S. Molecular Physics 1984, 52, 255.

(36) Hoover, W. G. Physical Review A 1985, 31, 1695.

(37) Ryckaert, J. P.; Ciccotti, G.; Berendsen, H. J. C. Journal of Computational Physics 1977, 23, 327.

(38) Essmann, U.; Perera, L.; Berkowitz, M. L.; Darden, T.; Lee, H.; Pedersen, L. G. Journal of Chemical Physics 1995, 103, 8577.

(39) Baez, L. A.; Clancy, P. In International Conference on Natural Gas Hydrates; Sloan, E. D., Happel, J., Hnatow, M. A., Eds.; New York Acad Sciences: New York, 1994; Vol. $715, \mathrm{p} 177$.

(40) Carver, T. J.; Drew, M. G. B.; Rodger, P. M. J. Chem. Soc.-Faraday Trans. 1996, $92,5029$.

(41) Carver, T. J.; Drew, M. G. B.; Rodger, P. M. In Gas Hydrates: Challenges for the Future; Holder, G. D., Bishnoi, P. R., Eds.; New York Acad Sciences: New York, 2000; Vol. 912, p 658.

(42) Storr, M. T.; Taylor, P. C.; Monfort, J. P.; Rodger, P. M. J. Am. Chem. Soc. 2004, $126,1569$.

(43) Westacott, R. E.; Rodger, P. M. Chem. Phys. Lett. 1996, 262, 47.

(44) MacKerell, A. D.; Bashford, D.; Bellott, M.; Dunbrack, R. L.; Evanseck, J. D.;

Field, M. J.; Fischer, S.; Gao, J.; Guo, H.; Ha, S.; Joseph-McCarthy, D.; Kuchnir, L.; Kuczera, 
K.; Lau, F. T. K.; Mattos, C.; Michnick, S.; Ngo, T.; Nguyen, D. T.; Prodhom, B.; Reiher, W. E.; Roux, B.; Schlenkrich, M.; Smith, J. C.; Stote, R.; Straub, J.; Watanabe, M.; WiorkiewiczKuczera, J.; Yin, D.; Karplus, M. Journal of Physical Chemistry B 1998, 102, 3586.

(45) Sloan, E. D.; Subramanian, S.; Matthews, P. N.; Lederhos, J. P.; Khokhar, A. A. Ind. Eng. Chem. Res. 1998, 37, 3124.

(46) Carver, T. J.; Drew, M. G. B.; Rodger, P. M. J. Chem. Soc.-Faraday Trans. 1995, $91,3449$.

(47) Fidler, J.; Rodger, P. M. J. Phys. Chem. B 1999, 103, 7695.

(48) Duffy, D. M.; Moon, C.; Rodger, P. M. Mol. Phys. 2004, 102, 203.

(49) Xia, T. K.; Landman, U. Science 1993, 261, 1310.

(50) Smith, P.; Lynden-Bell, R. M.; Smith, W. Molecular Physics 2000, 98, 255. 
Graphical Abstract
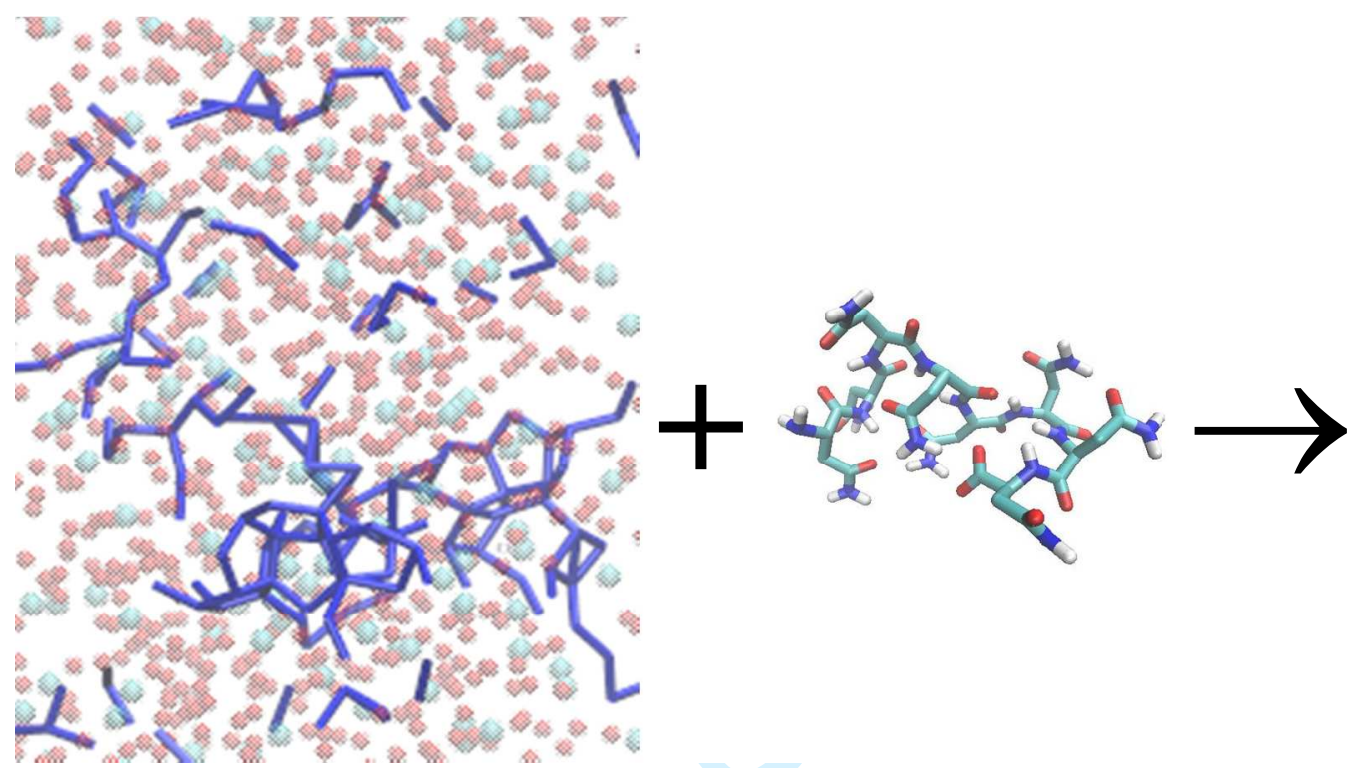

$?$ 